\title{
Methods for analyzing decision-making: a framework approach
}

\author{
Jon Nyhlén • Gustav Lidén
}

Published online: 23 July 2013

C Springer Science+Business Media Dordrecht 2013

\begin{abstract}
In political science an eternal question concerns how decisions and policies arise. Modern society, characterized by more uncertainty and complexity than before, increases the challenge of providing valid answers. However, the general lack of methodological concern in several previous studies in this area stresses the need for elaborations of more suitable approaches. In this article we add a methodological perspective that deals with this very question. By reviewing and analyzing earlier research on how decision-making is reached, the overall ambition of this article is to create a framework that can lay the methodological foundation for further studies. Such a framework, which takes into account both the complexity of modern multi-governance societies and adds methodological perspectives of macro and micro standpoints as well as of causal mechanisms, can be used in future research to achieve richer pictures of how decision-making is carried out. In addition, we show how a certain technique of analysis is highly compatible to this framework and that jointly these features provide solutions for a better understanding of the complexity of modern decision-making. All in all, applying this strategy can be used to better systematize complex causal chains that reflect different analytical levels and thereby increase leverage on how to understand and explain the process of political decisions.
\end{abstract}

Keywords Decision-making $\cdot$ Governance $\cdot$ Policy $\cdot$ Causal mechanism $\cdot$ Process tracing

\section{Introduction}

How political changes are brought about through processes of decisions has long been regarded as a key question in the disciplines that study the political dimensions of society. Several scholars have, though, noticed that drivers of political change today are less linear and harder to predict than previously (Held 2000; Pierre and Peters 2005). Often

J. Nyhlén · G. Lidén $(\varangle)$

Department of Social Sciences, Mid Sweden University,

Holmgatan 10, 85170 Sundsvall, Sweden

e-mail: gustav.liden@miun.se 
summarized by the term complexity, modern welfare states today face decision-making situations that are characterized by more of dynamism and uncertainty than before (Duit and Galaz 2008; Klijn 2008). Theoretical improvement has tried to capture this development. The perspective governance is evidence of that. A consequence of such a theoretical innovation has been an increase in micro-oriented empirical research (Verschuren and Arts 2005). However, the methodological consequences have not been fully implemented in line with such changes and are falling behind. Scholars in particularly public administration have been critical of the lack of concern about research design and method in the field, including both quantitative and qualitative techniques (Gill and Meier 2000; Lowery and Evans 2004). In relation to the empirical and theoretical changes mentioned, this article presents a way of trying to fill this lacuna. Therefore, focusing on decision-making, the purpose of this article is to create a unified methodological framework for analyzing the complexity in modern decision-making processes. In line with the purpose, this article will offer a perspective on the following research questions: how can processes of decision-making be analyzed in political systems? More concretely, this contribution will be presented in the form of a methodological framework and associated suitable techniques for analyses.

The introduction of system-theory that was initiated in political science during the 1960s was, in many ways, of great importance for political analyses (Deutsch 1963; Easton 1965), since it outlined a theoretically founded view of a political system. The greatest deficiency of this approach was, though, the fact that system-theory did not provide any tools for analyzing decision-making; rather, this was described as a "black box." Compared to these pioneer works, political systems of today are regarded as more complex (Jervis 1997; Klijn 2008) and also are characterized as being dynamic and self-organized. This is not only due to progress in science and improved abilities to detect such nuances, but is also necessarily so because of the dramatic changes political systems, especially in Western democracies, have undergone. Deriving from the classic words of Harold Lasswell, it is still true to say that politics is about who gets what, when, and how (Laswell 1935), and the instrumental view, attached to a political system, is thus to distribute and perhaps redistribute collective values. But in modern societies this task is in many ways much more challenging than before. In the words of Pierre and Peters (2005):

In the current perspective, the state evolves as an actor which remains in control of some unique power bases in society... At the same time, however, states are becoming increasingly dependent on other societal actors.

Pierre and Peters (2000) give three reasons as to why. First, modern welfare states sometimes lack the necessary resources for carrying out their commitments. Second, they can lack legitimacy, since other actors are assumed to fulfill some tasks more efficiently. Third, states face sectors in society which are becoming hard to govern. These challenges that political systems have to tackle also result in consequences for political science and for the study of decision-making as one of the core issues of the discipline. Many of the changes that the states of today face have been introduced through such ideas as new public management and policy networks. This has meant privatization, decentralization, and policy formation through networks and markets and has led to the traditional hierarchical model of government steering becoming challenged by a new style where steering is currently done in a complex environment with varying outcomes. In the foreground of the theoretical concepts that capture this described development, governance is found (Stoker 1998; Pierre and Peters 2000; Kooiman 2003; Kjær and Kjær 2004). Most fairly described as a paradigm, it cannot be considered especially bold to argue that governance prevails in research that focuses on decision-making processes in political systems. However, how decision-making should be 
analyzed when the point of departure is a concept that implies that various actors are involved in steering political development and thereby shaping political decision is still unclear.

This leads to a need to consider several questions related to the design of studies in this area. If modern political processes are complex and characterized by the participation of several different actors, what empirical material should be collected and how can it be analyzed to reconstruct and explain the causal chain of decision-making? The complexity increases even more if it is taken into consideration that all social processes are under the potential influence of both actors but also of structures founding society (Coleman 1990). Throughout this article, the methodological consequences of a more complex society and its impact on decision-making will be in focus. We thereby leave out much of the ongoing debate on how decision-making can be theorized and will limit our theoretical elaboration to the complexity of modern societies. In this study we show how a framework, based on a separation between actors and structure and inside or outside perspectives in relation to the system under study, can be helpful in analyzing political changes. In addition, we connect this framework with the technique of process tracing, arguing that this combination is a suitable way to reach leverage on how causal chains can be understood.

This article will begin with a closer look at the circumstances that characterize modern societies. This part is followed by a section in which the methodological challenges are discussed in a more general view. In the subsequent parts, answers to the research question are given by presenting methodological frameworks and techniques for the outlined ambition of this article.

\section{Theory and method for a changing society}

Addressing the formulated research question will make it necessary for this article to have two parts. When arguing that modern states are most correctly described as complex political systems, a theoretical elaboration of what this results in needs to be included. However, more important are the consequences that scholars in social sciences are facing when designing and carrying out their inquiries. Hence, perspectives having a background in theory of science will be discussed in relation to the distinction of structure and agency and ways to merge these aspects. With the focus being decision-making, an attempt will be made to separate out the surrounding conditions for political changes.

\subsection{Complexity in political processes}

In the Anglo-American literature, the term government has traditionally been used to refer to the formal institutions of the state and their monopoly over legitimate coercive power. Government is characterized by having a straightforward method about how decisions are made and enforced (Stoker 1998). As previously argued, this perspective has been challenged by the changes in the public sector, and complexity in public processes has continued to increase. The unclear boundaries between the political systems, their administrative subsystems, and external nonpublic actors are such examples (Kettl 2002). Much of this is a consequence of both the economic problems related to the modern welfare state and the ideological shift that meant the embracing of philosophies from the private market. The latter transformation, often communicated through the concept of new public management (Lane 2000) has, in a significant way, influenced political systems.

The uniting paradigm that is characterized by these changes is often presented through the concept of governance. Governance challenges the traditional view of the political systems 
and captures thereby many of the previously described trends (Kooiman 2003; Kjær and Kjær 2004). It refers to a complex set of actors and institutions that are drawn both from and beyond government (Stoker 1998). This means that in a governance structure policy is created both inside and outside the traditional hierarchical public sphere. Governance recognizes that policies are created and decisions are made in coalitions outside the traditional political system through various organizational forms such as policy networks and markets. It can be seen from theory emphasizing the complexity of contemporary governance processes that the idea of actors as the driving forces of political change as well as the need to take into account the context that they face, not least the institutional landscape, also result in important methodological consequences (Duit and Galaz 2008; Teisman and Klijn 2008). To increase clarity about these actors, research has tried to make distinctions about their origin and representation, but such attempts shows that such categories, in particular the one between private and public actors, are not self-evident (Lundqvist 1988). Since the traditional model of government is regarded as obsolete since it is not always a public agent that provides social services or other common goods. Creating markets where different actors compete with each other also creates a situation where actors will increase in numbers. In a market situation it is not only the citizens and the political sphere that are relevant. New actors need to be added, such as producers, clients, and customers.

Since governance most fairly must be described as a vague concept, it is constituted of different interpretations. One that harmonizes with the given perspective of political processes as complex is the one that characterizes governance as policy-making in networks (Rhodes 1996, 1997; Goodsell 2006; Provan and Kenis 2008). The central aspect of decision-making in networks is cooperation. The networks are usually described as self-organizing, where the actors come together and use each other's resources. The driving forces behind the networks are the different sets of resources. Rhodes (1997, p. 15) defines networks as "self-organizing, interorganizational networks characterized by interdependence, resource exchange, rules of the game and significant autonomy of the state." With this perspective, there is no monolithic power center. Instead, there are several centers from which steering is carried out (Rhodes 1997). In an interesting contribution, Wachhaus (2012) shows how networking governance is a way to anticipate a complex reality. He finds similarities between the ideological foundations that anarchy rests on and the characteristics of networks, namely decentralization, self-organization, interdependence, and horizontal organization. To conclude, such attributes are clearly measurements for better facing the changing circumstances of modern societies.

Although there are different interpretations of how steering and decision-making is carried out in contemporary societies, there are also several solutions. Pierre and Peters (2000) argue that the traditional hierarchical model for policy-making is still relevant. The only dependency that exists is then to the rules and the legal system. In this perspective the degree of flexibility in the model is limited. The bureaucratic principle of organization means that the government has full control over the means of production and its result. In this way, efficiency and full control are achieved. The bureaucracy then becomes an instrument to coordinate production and consumption of what is produced. Turning to decision-making, the early perspectives of how decisions are shaped (Simon 1957; Lindblom 1959) were contested in some important contributions. In accordance with the time, different scholars (Cohen et al. 1972; Kingdon 1984) opposed the earlier view and described the social system in which decisions were created as chaotic, unpredictable, and even anarchical. The now well-known alternative of visualizing such processes as independent streams in a system was believed to be a more appropriate way of how to capture a complex reality. Contemporary research stresses even more the complexity that includes the governance of and decision-making in modern societies that faces an appeal for interaction, networking, and participatory processes (Loorbach 2010). 
Disagreements clearly exist about how governance should be regarded, but that modern states have undergone significant changes must be regarded as generally accepted. New theoretical perspectives have tried to cope with new empirical circumstances. Nevertheless, updated methodological approaches for systematizing and analyzing data are absent. We will now turn to a discussion where such challenges are highlighted.

\subsection{Methodological perspectives on decision-making}

The methodological consequences of a study object characterized by governance, where steering involves actors from various political levels, where actors originate from both inside and outside the political system, and where different organizational forms jointly create decisions put a focus on how research in this field can be carried out. Hence, some more methodological perspectives on how to look at decision-making need to be discussed.

Analyzing decision-making is something of a core issue in political systems. Therefore, research in this field needs an understanding of how decisions are reached and needs to create valid theories that can predict such causal processes. A few criteria can be addressed. First, using a more methodological nomenclature, explanations of decision-making processes should address a holistic perspective, meaning that they ought not to be satisfied with identifying covariational patterns but should also try to explain how decisions are causally shaped (Lidén 2013). That is, reaching internal validity ought to be a goal as well as trying to increase accuracy in causal inference. Second, as has been stressed in the discussion above, various types of actors are involved in these types of processes. However, sometimes they are acting individually and sometimes groups of them are speaking with one voice, for example in the form of networks (Rhodes 1997). Research in this area needs to take this dynamic into account. Third, actors are always embedded in a milieu. They are, for example, influenced by norms, values, and cultures from surrounding societies. Differences in their spatial origin are relevant, i.e. derived from inside or outside political systems. These three criteria are crucial for reaching valid results and will guide this section.

In contemporary research in the social sciences, a growing belief is found in methodological individualism (Udehn 2002; Rothstein 2005, pp. 36-38). The reasons for applying these types of micro perspectives are often presented in a convincing way, for example: "The key word is 'individual'. In the social sciences, a satisfactory explanation must ultimately be anchored in hypotheses about individual behavior" (Elster 2007, p. 36). Such an approach, while not only representing a reasonable epistemological assumption, clearly harmonizes with the emphasis on actors in complex decision-making processes (Duit and Galaz 2008; Teisman and Klijn 2008). One of the most obvious methodological consequences of this renewed interest is a particular focus on causal mechanisms (Gerring 2010). The general idea with this concept is that the phenomena of causal mechanisms are the entities that connect explanans with explanandum in an explanatory model (Hedström and Swedberg 1998; Hedström 2005). A universal illustration of causal mechanisms is provided through the I-MO model where input (I) is linked with output $(\mathrm{O})$ through an intervening causal mechanism (M). The primary leverage that the introduction of the causal mechanism brings is that, potentially, an additional research question can be answered. More specifically, identifying not only the covariational patterns between explanans and explanandum, but also explaining how such a relationship is brought about, makes it possible to answer both "why" and "how."

There are some important consequences that follow from this. First, and already implied, a micro perspective is needed for analyzing causal mechanisms (Mahoney 2001; Gerring 2008, 2010) since this makes it possible to open the "black box" and allow a within type of analysis in which political changes arise (Gerring 2007). This is a development that corresponds to 


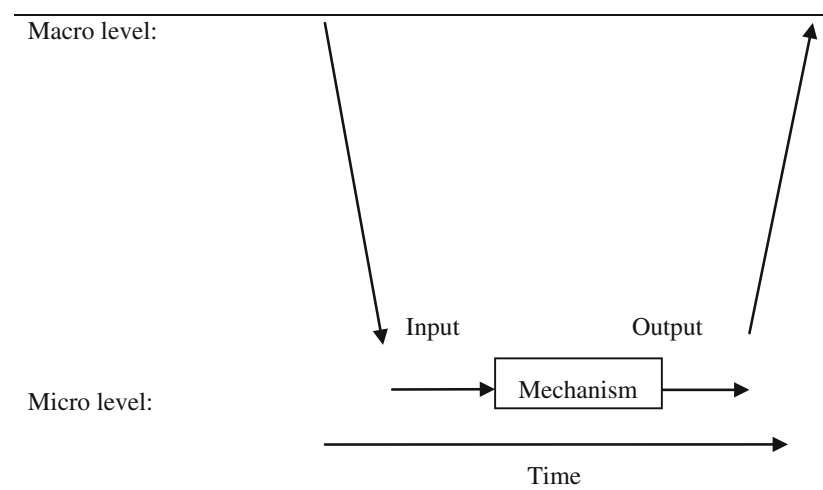

Fig. 1 Elaboration of the macro-micro-macro model

the theoretical shift from government to governance. To increase theoretical leverage either an inductive or deductive approach will need focusing on the actors that take part in shaping decisions. Further, and quite self-evidently, the order of time must be correctly specified. Assuring that the independent factor precedes the dependent one minimizes the potential problem of reverse causations and makes it possible to construct a chain of evidence (Peters 1998).

So far, and following from the first criterion formulated above, analyzing decision-making leads to a necessary interest in causal mechanisms. However, for the other two criteria to be reached another methodological dimension needs to be added. Discussing social phenomena from a spatial dimension makes it clear that they are not only exposed to a horizontal context but are also under the influence of a vertical one (Przeworski and Teune 1970, p. 12). An example will show that this is hardly surprising. Study a local councilor and it will be obvious that this actor will not only need to consider the opinion of his fellow party members but will also need to take into account policies, regulations, and ideas from international organizations, the central government, and his party organization on a national level before making a decision. Hence, this actor is part of a social system, and it is not only their motives or beliefs from "within" that characterize the actor's decision-making processes. In theory, the concept of multi-level governance is often used to depict such situations. But how does it work when this kind of structure influences decision-making? On a more abstract level, Coleman's (1990) famous model of the linkage between macro, micro, and macro levels is a suitable point of departure. In Fig. 1 this model has simply been merged with the I-M-O model discussed earlier, which makes it possible to simplify the relationship between analytical levels and simultaneously point out how mechanisms connect factors.

Some important consequences are shown in the figure. In line with the principles of methodological individualism, variables found on a macro level need to be related to certain actors or group of actors on micro levels. This is due to the fact that it is, in the end, the actor or actors that make decisions that have the potential to lead to political changes (Elster 2007). In this situation these decision-makers are potentially exposed to both macro and micro influences. Together with their own motives and beliefs this shapes their acting. Added to the traditional framework is the fact that the micro-micro linkage is carried out through causal mechanisms. Turning to the outcome of this model, it is not necessarily carried on a macro level, though it can be. For that to happen, several actors need to interact and jointly create a collective outcome that can be positioned, analytically, on a macro level. In the case of 
political decision-making this can, for example, be a new regulation that is implemented by a group or institution in society.

To conclude, in analyzing decision-making in political systems, not only the explanatory variable but also the causal mechanisms that lead to a decision need to be studied in connection with the fact that specific actors, or groups of actors, are those who make decisions and that they, potentially, are influenced by circumstances from both macro and micro levels. If such a point of departure is taken into consideration when analyzing decision-making, the previously discussed criteria can be reached and the validity potentially increases.

\subsection{Combining theory and method}

So how can these perspectives be used when analyzing processes of decision-making? The paradigm of governance is not a unified theoretical construction, but it does point to several important notions that need to be taken into account. First, decision-making processes in a governance context are recognized as complex and as driven by actors. Modern governing includes actors and institutions both from within and from outside the formal political organization (Stoker 1998). This implies a micro perspective (Verschuren and Arts 2005). Second, governing today includes many actors and institutions with different spatial origins. The methodological discussion supports this idea, arguing that an interesting relation between macro and micro levels exists. Third, since these processes are complex, understandings and explanations of the decision-making process should strive for models that include both correlations and causal mechanisms (Lidén 2013) and thereby increase precision and validity.

\section{A framework for analyzing decision-making}

Taken together, the discussed descriptions of modern society connected with improvements in the methodological area have given valuable contributions about how to look upon the process of decision-making. The essence of the theory of governance reflects a complex governing structure where, potentially, groups are influencing the outcome of decisions while originating from different spatial positions and representing different interests (Stoker 1998; Kettl 2002). The main feature of the methodological discussion is that both macro and micro levels need to be taken into account when analyzing decision-making so that the threads that connect input with output can be explained. In sum, we have on the one hand an empirical reality that is complex, where both actors and structures influence decision-making and, on the other hand, methodological claims that stress the need for systematizing this reality so that it is possible to analyze it. Clearly, some sort of framework for making this possible must be regarded as crucial.

To reach this, two dimensions can be distinguished. First of all, and in relation to the paradigm of methodological individualism, structural conditions can be separated from actorsoriented explanations. In analogy with both Elster (2007) and the scholars that emphasize the need for examining causal mechanisms (Hedström and Swedberg 1998; Mahoney 2001; Gerring 2008), structures are regarded as potential influences on individuals' behavior and thereby decision-making; however, isolated they cannot cause political change (Coleman 1990). Both this and the second dimension are ways of coping with the spatial variation established in a complex society. Turning to the second dimension, it implies a distinction between actors' and structures' origin from either inside or outside the system where the decision-making is carried out. Thus, what constitutes the actual systems is defined in 
Table 1 A framework for analyzing decision-making

\begin{tabular}{lll}
\hline & Structural conditions & Actors-oriented explanations \\
\hline Exogenous & Exogenous structural conditions & Exogenous actors-oriented explanations \\
Endogenous & Endogenous structural conditions & Endogenous actors-oriented explanations \\
\hline
\end{tabular}

the design of each separate study. Really, this distinction can be regarded as obsolete as contemporary theories have emphasized that political decisions are reached between actors representing different spatial origins (Kettl 2002). However, it does have analytical strengths. By making an analytical separation between endogenous and exogenous origin, analytical leverage is increased (e.g. Lidén 2011) and the theoretical contribution will be easier to systematize. The framework is presented in Table 1.

Exogenous structural conditions are characteristics of a whole society. These variables are frequent in the aggregated research that, for example, is represented by comparative politics or comparative-historical analysis. Such macro variables are important when constructing a chain of evidence. Structural conditions from the inside can also influence the process of decision-making. These are traits that, for example, reflect cultures, norms, and values that characterize a system. However, they can also indicate hard data, such as the internal economic conditions of an organization. A perhaps more concrete example is the influencing factor an organizational culture (Schein 1990) could have on decisions shaped at an agency or local council.

Turning to the right hand side of the typology, in the traditional view of a political system, citizens and other actors from the surrounding environment demand implementation of certain policies (Easton 1965). In line with the paradigm of governance, important actors can be expected to be found here. Finally, the beliefs and convictions of actors inside the political systems are crucial sources for the process of decision-making. When explicitly referring to political systems, these key actors are either politicians or civil servants.

By giving an invented empirical example the potential usage of this framework will be more transparent. Imagine a political system consisting of a municipality. The boundaries are in this example quite clear, since the municipality represents an own political system with its own demos. Endogenous conditions and explanations are positioned within the organization of the municipality. Actors in such a system are thus either employees of the local government or politicians active in the local democracy. Exogenous conditions and potential explanations of decision-making are derived from the surrounding local society. Picture the following scenario. A few citizens of our municipality are displeased with the function of the local democracy. They feel ignored by the local political elite which they mean has decoupled the surrounding society. Hence, we can talk about a democratic deficit (Papadopoulos 2003). The factors that can cope with this problem can originate from different parts of a social system. Deriving from resource theory, we know that citizens with a lot of political resources (Verba et al. 1995) are more inclined to participate. Thereby, structures in society such as a high level of income and education will increase the potential that displeased citizens will contact leading politicians and convey their displeasure, especially so when dissatisfaction with the local democracy is another form of societal structure. In short, specific structural conditions will increase the chances of citizens influencing the political society; see Table 2 for steps 1-3 in the given example. The emergence of such ideas is, though, dependent on certain groups of citizens that, isolated or together with others, introduce this through gatekeepers, as has previously been described by Easton (1965). When this input is received by the political system, actors, such as the political elite, are equipped with the mandate for creating political 
Table 2 Exemplifying flows in the framework

\begin{tabular}{|c|c|c|}
\hline & Structural conditions & Actors-oriented explanations \\
\hline Exogenous & 1. Exogenous structural conditions & $\begin{array}{c}\text { 2. Exogenous actors-oriented } \\
\text { explanations }\end{array}$ \\
\hline Endogenous & 4. Endogenous structural conditions & $\begin{array}{l}\longrightarrow \\
\text { 3. Endogenous actors-oriented } \\
\text { explanations }\end{array}$ \\
\hline
\end{tabular}

change. Of course their own beliefs and opinions from "within" are important. In this case, this reflects citizens' participation in public discussions (Fung 2006). However, as indicated above, there are also endogenous structural conditions that need to be taken into account. In this example, measurements for better involvement of citizens in the democratic process can result in different outcomes: local referenda or e-democracy citizen juries. However, as shown in the table, conditions for making decisions are not only due to the input from the surrounding society or the motives and beliefs of the decisions-makers but are also dependent on structural conditions inside the political systems, in this example the municipality. This is illustrated by the arrow from step 4 to 3 in Table 2. Economic constraints as well as the dominating visions, culture, and ideas inside an organization delimit possible decisions. Formally, official political decisions are taken by specific political leaders, but as shown by the outline they are facing different types of influences. To conclude, a possible description of the processes of decision-making has been presented, indicating how exogenous and endogenous influences with either a structural or an individual background can interplay. The presented flows should be regarded as conceivable but not at all as exclusively towards other causal chains.

Obviously, this typology does not fairly reflect the various conditions and actors that influence and explain decision-making. First of all, there exist many possible situations where borderline cases can occur. In fact, the outlined complexity is characterized by unclear boundaries and a heterogeneity in the included actors' origin and characteristics. In the given example these are handled in a simplified way. Second, the fact that these different types of influences are interrelated as a complex chain of explanations gives a truer picture. More concretely, combinations of influencing conditions and explanations can be understood more easily when working with such a framework. To conclude, the advantages of analytical frameworks, such as the one above, gain in strength initially when they are connected to either theoretical or methodological approaches. Since the presented framework is based on a meta-theoretical perspective of modeling how decisions can be created, we therefore turn to discuss some analytical techniques.

\subsection{Analytical strategy}

Several criteria have been mentioned above that are about how to create valid social science when deriving from a complex social system. Transforming such notions to concrete techniques for social inquiry will never result in a catch-all list. Hence, this section will 
discuss one strategy, that we in particular find suitable and that adds combinatory values when adapting it to the described framework.

As one of several outcomes of the recent intense debate on research design carried out in political science (King et al. 1994; Brady and Collier 2004; Brady 2006; Beck 2010; Collier et al. 2010), the concepts of causal mechanisms and micro foundations have been repeatedly discussed. They have been described as almost an obsession of contemporary social science (Gerring 2010), but this approach has also resulted in several advantages. Many of the demands that this scope requires have, appropriately, been argued to be fulfilled if working with process tracing (Tarrow 2004; George and Bennett 2005; Gerring 2007; Beach and Pedersen 2013). This form of analysis derives from one or a few cases and has the ambition of reconstructing the causal chain, including the mechanisms, which leads forward to an influence on and a change of the dependent variable. Among the particular characteristics of process tracing is the need to reach a detailed order in which different types of data, e.g. data triangulation (Tansey 2007; Yin 2009), are included and where the time span is often considerable. As formulated by Coppedge (1999), this type of extended case study has the potential to reach good validity when working with complex concepts that are extracted from modern political systems. Another strength of process tracing is the narrow focus and the potential of counterfactual reasoning that gives measures for handling this potential bias (King et al. 1994; George and Bennett 2005). Although process tracing has not been too frequently used when analyzing political processes of decision-making, this approach has a considerable history in other disciplines such as psychology, economics, and organizational theory (Payne 1976; Todd and Benbasat 1987).

The theoretical implications of process tracing are disputed (King et al. 1994; e.g. Munck 2004). The traditional argument, that case studies are more appropriate in developing than testing theories, must be regarded as still prevailing. Another reason for this statement is more unsatisfying and derives from the fact that theoretical descriptions seldom include any information of detailed causal mechanisms. However, case studies working with process tracing still have the advantage of collecting data with broad time frames and thereby also testing theories where other methods fail (Coppedge 1999). Nevertheless, scholars applying process tracing often work more inductively even if this can sometimes include constructing theoretical leverage by increasing the precision of an established theory by filling in the links between the independent and dependent factor. The possibility of causal inference based on a small-N design is, though, limited (King et al. 1994).

Complex political processes clearly include many factors that potentially influence the outcome of decision-making. The analytical problems increase due to the fact that these factors can interact with each other. We argue here that process tracing, together with the discussed framework, form a valuable approach. Scholars often depart from theoretical constructions that can be helpful in the systematizing and ordering of causal pathways. Thus, the real advantage of process tracing is revealed when constructing valid frameworks for ordering and connecting the relevant events of a development process. As shown by Mahoney (1999), the value of collected data can be increased by using the logic from process tracing. The generic framework presented above represents another strategy that can be the point of departure for a causal analysis. This framework allows for outlining a scheme that visualizes the potential interplay between structure and agency as well as exogenous and endogenous. It is still theoretically founded, but in this case on a meta-theoretical perspective including an ontological view on how political change is brought about (Marsh 2010). Using the discussed approach makes it possible to identify and examine the crucial interaction between structural conditions and political change achieved by certain actors but also to trace whether such conditions or explanations arise inside or outside the studied system. Such deep studies, 
which make it possible to work with different type of empirical material, potentially increase the accuracy of causal links.

\section{Conclusions}

As argued in the introduction, this article presents a way of how to look at the decisionmaking process that results from a complex society. The discussed theoretical dimensions are examples of developments that are consistent with the reality that political science studies. It recognizes the complex governance of modern societies (Pierre and Peters 2005). However, the absence of the methodological consequences of this has been in focus for this study (Gill and Meier 2000; Lowery and Evans 2004). Important conclusions can be drawn from this, and we now turn to discuss three topics that are found to be of certain importance.

First, this article adheres to the demand for intensified concern for research design and methodological development in the field analyzing political change. Of course, better designed studies utilizing state-of-the-art methods will increase the impact of research when scientific conclusions can be transformed into policy. An ongoing discussion of design and method, considering the full spectra from ontological to practical epistemological questions, is important and also necessary to create prerequisites for such a development (King et al. 1994; Brady and Collier 2004).

Second, the proposed framework gains relevance if it is combined with theoretical and methodological subsets. The theoretical perspectives that can be embedded in this framework are not specified in this study since they cannot easily be limited to any definite category. A vast group of theories in social science provide hypotheses of political change. We have instead focused on the discussion of appropriate techniques for analyzing decision-making. By combining the in-depth focus of process tracing and a framework that includes relevant elements for explaining the social world, this can be achieved. However, the crucial part is to combine an analytical framework with a methodological strategy since that creates possibilities to efficiently analyze the material. Ordering the material into the different fields of the typology and connecting different parts of them to each other with the use of process tracing increase the potential validity (Mahoney 1999).

Third, in line with the purpose of this article, the framework that has been reached offers an analytical solution to how decision-making can be explained. In that sense, this contribution is different from traditional explanatory theories. In other words, it offers no idea of how phenomena in the social world are connected. Instead, the discussed framework and process tracing as a suitable technique give direction to an approach on how to embed relevant theories. The consequences of this strategy can be used to better systematize complex causal chains, reflecting different analytical levels when it comes to understanding and, if possible, explaining how decisions are made.

To conclude and relate to the purpose of this article, the study has shown one way of how the complexity of decision-making can be understood more easily. Although a method for analyses and a strategy of how data can be collected and ordered, there are several other parts of a research process that are crucial. Working with this approach also makes it necessary to, either inductively or deductively, connect it to theory and also to assemble material, rich in both quality and quantity, which makes it possible to offer explanations of decision-making in a more valid way.

Acknowledgments An earlier draft of this article was presented at the Twentieth NorKom Conference, University of Gothenburg, Gothenburg, November 24-26 2011. We are grateful to the participants for valuable comments. 


\section{References}

Beach, D., Pedersen, R.B.: Process-Tracing Methods?: Foundations and Guidelines. University of Michigan Press, Ann Arbor (2013)

Beck, N.: Causal process "observation": oxymoron or (fine) old wine. Political Anal. 18, 499-505 (2010)

Brady, H.E.: Toward a pluralistic vision of methodology. Political Anal. 14, 353-368 (2006)

Brady, H.E., Collier, D. (eds.): Rethinking Social Inquiry?: Diverse Tools. Shared Standards. Rowman \& Rowman, Lanham (2004)

Cohen, M.D., March, J.G., Olsen, J.P.: A garbage can model of organizational choice. Adm. Sci. Q. 17, 1-25 (1972)

Coleman, J.S.: Foundations of Social Theory. Harvard University Press, Cambridge (1990)

Collier, D., Brady, H.E., Seawright, J.: Outdated views of qualitative methods: time to move on. Political Anal. 18, 506-513 (2010)

Coppedge, M.: Thickening thin concepts and theories: combining large $\mathrm{N}$ and small in comparative politics. Comp. Politics 31, 465-476 (1999)

Deutsch, K.W.: The Nerves of Government?: Models of Political Communication and Control. Free Press, New York (1963)

Duit, A., Galaz, V.: Governance and complexity-emerging issues for governance theory. Governance 21, 311-335 (2008)

Easton, D.: A Framework for Political Analysis. Prentice-Hall, Englewood Cliffs (1965)

Elster, J.: Explaining Social Behavior?: More Nuts and Bolts for the Social Sciences. Cambridge University Press, Cambridge (2007)

Fung, A.: Varieties of participation in complex governance. Public Adm. Rev. 66, 66-75 (2006)

George, A.L., Bennett, A.: Case Studies and Theory Development in the Social Sciences. MIT Press, Cambridge (2005)

Gerring, J.: Case Study Research?: Principles and Practices. Cambridge University Press, New York (2007)

Gerring, J.: The mechanismic worldview: thinking inside the box. Br. J. Political Sci. 38, 161-179 (2008)

Gerring, J.: Causal mechanisms: yes, but... Comp. Political Stud. 43, 1499-1526 (2010)

Gill, J., Meier, K.J.: Public administration research and practice: a methodological manifesto. J. Public Adm. Res. Theory 10, 157-199 (2000)

Goodsell, C.T.: A new vision for public administration. Public Adm. Rev. 66, 623-635 (2006)

Hedström, P.: Dissecting the Social?: On the Principles of Analytical Sociology. Cambridge University Press, Cambridge (2005)

Hedström, P., Swedberg, R.: Social Mechanisms?: An Analytical Approach to Social Theory. Cambridge University Press, Cambridge (1998)

Held, D.: Regulating globalization? The reinvention of politics. Int. Sociol. 15, 394-408 (2000)

Jervis, R.: System Effects?: Complexity in Political and Social Life. Princeton University Press, Princeton (1997)

Kettl, D.F.: The Transformation of Governance: Public Administration for Twenty-First Century America. JHU Press, Baltimore (2002)

King, G., Keohane, R.O., Verba, S.: Designing Social Inquiry?: Scientific Inference in Qualitative Research. Princeton University Press, Princeton (1994)

Kingdon, J.W.: Agendas, Alternatives, and Public Policies. Foresman and Company, London (1984)

Kjær, A.M., Kjær, A.M.: Governance. Polity Press, Cambridge (2004)

Klijn, E.-H.: Complexity theory and public administration: what's new? Public Manag. Rev. 10, 299-317 (2008)

Kooiman, J.: Governing as Governance. Sage, London (2003)

Lane, J.E.: New Public Management. Routledge, London (2000)

Laswell, H.D.: Politics: Who Gets What?. University of Chicago Press, Chicago (1935)

Lidén, G.: Democracy and Diffusion Creating a Comprehensive Model for Explaining Democracy. Mittuniversitetet, Sundsvall, Inst. för samhällvetenskap (2011)

Lidén, G.: What about theory? The consequences on a widened perspective of social theory. Qual. Quant. 47, 213-225 (2013)

Lindblom, C.E.: The science of “muddling through”. Public Adm. Rev. 19, 79-88 (1959)

Loorbach, D.: Transition management for sustainable development: a prescriptive, complexity-based governance framework. Governance 23, 161-183 (2010)

Lowery, D., Evans, K.G.: The iron cage of methodology. Adm. Soc. 36, 306-327 (2004)

Lundqvist, L.J.: Privatization: towards a concept for comparative policy analysis. J. Public Policy 8, 1-19 (1988) 
Mahoney, J.: Nominal, ordinal, and narrative appraisal in macrocausal analysis. Am. J. Sociol. 104, 1154-1196 (1999)

Mahoney, J.: Beyond correlational analysis: recent innovations in theory and method. Sociol. Forum. 16, 575-593 (2001)

Marsh, D.: Meta-theoretical issues. In: Marsh, D., Stoker, G. (eds.) Theory and Methods in Political Science. Palgrave Macmillan, New York (2010)

Munck, G.L.: Tools for qualitative research. In: Brady, H.E., Collier, D. (eds.) Rethinking Social Inquiry: Diverse Tools, Shared Standards, pp. 105-121. Rowman \& Littlefield, Lanham (2004)

Papadopoulos, Y.: Cooperative forms of governance: problems of democratic accountability in complex environments. Eur. J. Political Res. 42, 473-501 (2003)

Payne, J.W.: Task complexity and contingent processing in decision making: an information search and protocol analysis. Organ. Behav. Hum. Perform. 16, 366-387 (1976)

Peters, B.G.: Comparative Politics: Theory and Methods. Macmillan, London (1998)

Pierre, J., Peters, B.G.: Governance, Politics, and the State. St. Martin's Press, New York (2000)

Pierre, J., Peters, B.G.: Governing Complex Societies?: Trajectories and Scenarios. Palgrave MacMillan, Basingstoke (2005)

Provan, K.G., Kenis, P.: Modes of network governance: structure, management, and effectiveness. J. Public Adm. Res. Theory 18, 229-252 (2008)

Przeworski, A., Teune, H.: The Logic of Comparative Social Inquiry. Wiley-Interscience, New York (1970)

Rhodes, R.A.W.: The new governance: governing without government. Political Stud. Lond. 44, 652-667 (1996)

Rhodes, R.A.W.: Understanding Governance?: Policy Networks, Governance, Reflexivity and Accountability. Open University Press, Buckingham (1997)

Rothstein, B.: Social Traps and the Problem of Trust. Cambridge University Press, Cambridge (2005)

Schein, E.H.: Organizational culture. Am. Psychol. 45, 109-119 (1990)

Simon, H.A.: Administrative Behavior?: A Study of Decision-Making Processes in Administrative Organization. Macmillan, New York (1957)

Stoker, G.: Governance as theory: five propositions. Int. Soc. Sci. Rev. 50, 17-28 (1998)

Tansey, O.: Process tracing and elite interviewing: a case for non-probability sampling. Political Sci. 40, 765-772 (2007)

Tarrow, S.: Bridging the quantitative-qualitative divide. In: Brady, H.E., Collier, D. (eds.) Rethinking Social Inquiry: Diverse Tools, Shared Standards, pp. 171-180. Rowman \& Littlefield, Lanham (2004)

Teisman, G.R., Klijn, E.-H.: Complexity theory and public management. Public Manag. Rev. 10, 287-297 (2008)

Todd, P., Benbasat, I.: Process tracing methods in decision support systems research: exploring the black box. MIS Q. 11, 493-512 (1987)

Udehn, L.: The changing face of methodological individualism. Annu. Rev. Sociol. 28, 479-507 (2002)

Wachhaus, T.A.: Anarchy as a model for network governance. Public Adm. Rev. 72, 33-42 (2012)

Verba, S., Schlozman, K.L., Brady, H.E.: Voice and Equality: Civic Voluntarism in American Politics. Harvard University Press, Cambridge (1995)

Verschuren, P., Arts, B.: Quantifying influence in complex decision making by means of paired comparisons. Qual. Quant. 38, 495-516 (2005)

Yin, R.K.: Case Study Research: Design and Methods. Sage, Thousand Oaks (2009) 